\title{
Influence of the Correlation Model on the Failure Probability of a Reinforced Concrete Structure Considering Spatial Variability
}

\author{
Eline Vereecken ${ }^{\mathrm{a}, \mathrm{b}, *}$, Wouter Botte ${ }^{\mathrm{a}}$, Geert Lombaert $^{\mathrm{b}}$, Robby Caspeele $^{\mathrm{a}}$ \\ ${ }^{a}$ Department of Structural Engineering and Building Materials, Ghent University, Ghent, Belgium \\ ${ }^{\mathrm{b}}$ Department of civil engineering, KU Leuven, Leuven, Belgium \\ *Corresponding author at Technologiepark-Zwijnaarde 60, 9052 Ghent, Belgium; eivereec.vereecken@ugent.be
}

\begin{abstract}
Reinforced concrete structures are often subjected to corrosion, inducing a decreasing reliability over time. This corrosion often exhibits spatial variation, which can be modelled by random fields. To improve the accuracy of the estimated reliability, measurements can be performed. In this work, focus will be on static strain data. Taking into account these measurements leads to updated distributions of the corrosion parameters and an updated reliability. However, modelling errors will arise due to an inadequate choice of the spatial correlation model. These are present since the perfect model is not known. By assuming an inaccurate correlation model, a bias on the distribution of the reliability index might arise. In this work, the influence of this modelling error is investigated by assuming a different correlation model in the calibration of the model and the generation of the data. Different correlation models are considered and the most robust spatial correlation model is searched for. This problem is considered first for a simply supported beam subjected to corrosion, with the aim of identifying the most robust model. Next, it is investigated whether this model also performs well for a bridge structure.
\end{abstract}

Keywords: Random fields, corrosion, Bayesian updating, correlation, concrete, RC bridge, static strain data, spatial variation

\section{INTRODUCTION}

Steel reinforcement bars in reinforced concrete (RC) structures are often susceptible to corrosion in aggressive environments. This corrosion process can reduce the resistance of the structure and hence influence its safety level. To gain insight in the corrosion process, different models exist to predict the influence of corrosion on the structural resistance. Enright \& Frangopol (1999) apply a resistance degradation function to calculate the evolution in time of the remaining fraction of the resistance. Faroz, Pujari, \& Ghosh (2016) calculate the remaining reinforcement area based on the steel material loss and a spatial area heterogeneity factor. Samarakoon \& Sælensminde (2015) calculate the remaining reinforcement area based on the probability of indication of active corrosion and the probability of corrosion correctly indicated by an inspection method. Stewart \& Rosowsky (1998) calculate the reduction in reinforcement diameter as a function of the time after initiation, and the corrosion rate, where the latter is determined from field or experimental studies. In addition, the effect of highly localised pitting is taken into account. The referred models often do not account for the spatial variation of the corrosion process. Although this spatial character of deterioration is discussed in for example (Hajializadeh, Stewart, Enright, \& OBrien, 2015; Stewart, 2006; Stewart \& Suo, 2009), information on this topic is still scarce. In addition, there is no commonly agreed approach on how to model this spatial character of the corrosion process. One way to model this spatial behaviour is by the application of random fields. However, different correlation models can be used and, for a given correlation model, different correlation lengths can be considered. All these assumptions will affect the modelled structural resistance and the corresponding probability of failure.

To gain more insight in the actual deterioration state of an RC structure, measurements can be performed. The resulting data can be used to update the variables used in the corrosion model by application of Bayesian updating techniques. The resulting posterior distributions are affected by the prior assumptions. An important assumption herein is the correlation model for the random field that is used to model the corrosion variables. If this does not adequately characterize the actual corrosion pattern, an important modelling error can be present, resulting in a biased posterior distribution that might still deviate significantly from the actual corrosion degree. In a conventional Bayesian updating approach, the prior distribution of a set of unknown variables $\theta$ is updated to a posterior distribution based on the available information. However, when multiple datasets are available from different experiments, these provide "alternative" information on the structure. When these multiple observations from a single system or component are used to characterize uncertainties, hierarchical Bayesian updating

Postprint submitted to Structure and Infrastructure Engineering

Published version: Vereecken, E., Botte, W., Lombaert, G., \& Caspeele, R. (2021). Influence of the Correlation Model on the Failure Probability of a Reinforced Concrete Structure Considering Spatial Variability. Structure and Infrastructure Engineering https://doi.org/10.1080/15732479.2021.1953082 
(Behmanesh, Moaveni, Lombaert, \& Papadimitriou, 2015; Gelman, Carlin, Stern, \& Rubin, 2004) can be used instead of a standard Bayesian approach.

In the case of hierarchical Bayesian updating, the different datasets are treated as the components or members of a population. When considered independently, each dataset will lead to different parameters $\mu_{\theta}$ and $\sum_{\theta}$ of the variables $\boldsymbol{\theta}$ in the model characterizing the structure. These parameter values $\mu_{\theta}$ and $\sum_{\theta}$ can therefore be thought of as samples resulting from a distribution for the parameters of $\boldsymbol{\theta}$ (denoted as $\boldsymbol{\mu} \boldsymbol{\theta}$ and $\sum \boldsymbol{\theta}$ ). In hierarchical Bayesian updating, the different datasets are used to identify this distribution of the parameters of the variables $\boldsymbol{\theta}$. This involves the calculation of a posterior distribution of the distribution parameters $\boldsymbol{\mu}_{\boldsymbol{\theta}}$ and $\sum \boldsymbol{\theta}$. Hence, in hierarchical Bayesian updating, the parameters of the variables of interest are modelled as uncertain themselves and these parameters are updated based on the available data. The more data becomes available, the smaller the posterior uncertainty. Hierarchical Bayesian updating starts from the principle that the posterior distribution of the variables of interest $\boldsymbol{\theta}$ is derived for each of the $N$ available datasets. Hence, $N$ different posterior distributions for $\boldsymbol{\theta}$ are found. Based on these posterior distributions, a posterior distribution for the parameters of $\boldsymbol{\theta}\left(\boldsymbol{\mu}_{\boldsymbol{\theta}}\right.$ and $\sum \theta$ ) can be derived. By modelling the parameters of the variables themselves $\left(\boldsymbol{\mu}_{\boldsymbol{\theta}}\right.$ and $\left.\sum \boldsymbol{\theta}\right)$ as uncertain, the reliability index will also be uncertain (Der Kiureghian, 2008). By updating the parameters of the variables in the corrosion model, the corresponding distribution of the reliability index will be updated as well.

In this work, a case study of an RC structure subjected to corrosion is considered. The available data sets consist of sampled strain measurements under proof-loading. It is investigated how the correlation model of the corrosion variables affects the distribution of the reliability index. Hierarchical Bayesian updating is applied to update the distributions of the parameters (mean and standard deviation) of the corrosion variables, accounting for strain measurement data. In order to investigate the influence of different actual correlation models, measurement results have been generated by simulation using a finite element model, in this way relating the strain response of the structure to the corrosion variables. To these finite element results, a (random) measurement error is added to generate the measurement data. As such, measurement results are generated corresponding to different underlying correlation models of the corrosion variables. For each of the simulated measurement results, the posterior distributions of the parameters of the corrosion variables are derived, considering different prior random fields. In this way, a possible prior modelling error is considered, where the assumed random field does not correspond with the random field model related to the data. As such, the influence of the prior assumption of the correlation model on the distribution of the reliability index is investigated. It should be pointed out that model errors will always be present in practice. These model errors can be accounted for in different ways. For example, in (Simoen, Papadimitriou, \& Lombaert, 2013) the correlation model of the model error to be used in the likelihood function when applying Bayesian updating is identified based on model class selection.

The outline of this paper is as follows. First, the methodology for the hierarchical Bayesian updating is explained. Next, the considered correlation models as found in relevant literature on corrosion are summarized. In section 4 , an example is introduced and in section 5 , the results of the posterior reliability indices are provided. Finally, in section 6 , a case study is considered.

\section{HIERARCHICAL UPDATING FRAMEWORK}

In the hierarchical Bayesian framework, the parameters of the variables of interest (i.e. their mean and variance) are updated instead of the variables themselves. In this work, the initiation period $\left(T_{i}\right)$ and corrosion rate $\left(V_{\text {corr }}\right)$ are the variables in the corrosion model and are modelled by random fields. The random fields are discretized by subdividing the structure in elements and the mean and variance of the initiation period and corrosion rate in each of these elements are modelled as uncertain. With the use of hierarchical Bayesian updating, the distributions of the mean and variance of the initiation period and the corrosion rate are updated. It should be pointed out that the considered elements in the discretization of the random fields do not necessarily coincide with the finite elements in the finite element model. The elements of the random fields can be larger than the finite elements and can contain different smaller finite elements. The midpoint approach is applied with element-wise constant values in the elements in the discretization of the random fields (Sudret \& Kiureghian, 2000). The discretization is chosen as such that convergence of the reliability index is achieved. In this work, the finite element model of the structure is constructed from different line, area and/or volume elements, with a size matching the discretization of the random field. Properties are assigned to these lines/areas/volumes according to the sample of the discretized random field. These lines/areas/volumes are then further subdivided in finite elements for construction of the finite element model.

To perform hierarchical Bayesian updating, the Gibbs sampler can be used (Geman \& Geman, 1984). However, this requires a lot of computational effort, since in this Gibbs sampling for the generation of one sample of the posterior variable $\boldsymbol{\theta}$ " (in this case the posterior of $T_{i}$ and $V_{\text {corr }}$ in all elements of the structure), a Markov Chain with Metropolis-Hastings algorithm needs to be run. In order to overcome this, the posterior distributions can also be obtained based on conjugate priors and Maximum A Posteriori (MAP) estimates. This approximate method is based on (Behmanesh et al., 2015) where stiffness distributions are updated based on available vibration 
data. According to (Beck \& Katafygiotis, 1998; Papadimitriou, Beck, \& Katafygiotis, 1997), the posterior PDF can be asymptotically approximated by a normal distribution, centred around the maximum a posteriori (MAP) point and with a covariance matrix $\widehat{\boldsymbol{\Sigma}}_{p o}$. For a set of parameters $\boldsymbol{\theta}$ (in this case the initiation period $T_{i}$ and corrosion rate $V_{\text {corr }}$ in all elements of the structure) with prior probability $f^{\prime}(\boldsymbol{\theta})$ the MAP point can be estimated from Equation (1).

$$
\widehat{\boldsymbol{\vartheta}}^{M A P}=\underset{\boldsymbol{\vartheta}}{\operatorname{argmin}}\left(F_{M A P}\right) \text { with } F_{M A P}=-\log (L(\boldsymbol{\vartheta} \mid \overline{\boldsymbol{d}}))-\log \left(f^{\prime}(\boldsymbol{\vartheta})\right)=F_{M L}+F_{M A P r}
$$

Here, $L(\boldsymbol{\vartheta} \mid \overline{\boldsymbol{d}})$ is the likelihood function accounting for data $\overline{\boldsymbol{d}}$. When this likelihood function is Gaussian, the first term $F_{M L}$ corresponds to a generalized least squares objective function, where the subscript $M L$ stands for Maximum Likelihood. The term $F_{M A P r}$ then corresponds to a regularization term based on the available prior information.

The approximate posterior covariance matrix $\widehat{\boldsymbol{\Sigma}}_{p o}$ is computed as the inverse Hessian of the MAP objective function $F_{M A P}$, evaluated at the MAP point, or by application of Equation (2):

$$
\widehat{\boldsymbol{\Sigma}}_{p o}^{-1}=\left.\nabla_{\boldsymbol{\vartheta}}^{2} F_{M A P}\right|_{\vartheta=\widehat{\vartheta}^{M A P}}
$$

In many optimization algorithms that solve for the MAP point, this Hessian is computed as a by-product in the solution of this optimization problem (Björk, 1996; Ipsen, 2009).

For a linear model and a Gaussian prior and prediction error model, the asymptotic approximation becomes exact. However, when the corrosion variables are to be updated based on strain data, the relationship between the corrosion variables and the measurement data is nonlinear. Hence, in this case non-linear least square methods are required to solve the optimization problem for the MAP point. Non-linear least-squares optimization finds a solution for problems of the type given by:

$$
\min _{a} 0.5 \sum_{i}\left(f_{i}(a)\right)^{2}
$$

If the likelihood is Gaussian, $F_{M L}$ can be written according to Equation (4), when disregarding the constant terms:

$$
F_{M L}=\sum_{i}^{n} \frac{\left(\bar{y}_{i}-y(\boldsymbol{\vartheta})\right)^{2}}{\sigma^{2}}
$$

Here, $\bar{y}_{i}$ is measurement result $i$ (i.e. measured strain at one sensor location), $y(\boldsymbol{\vartheta})$ the model that relates the uncertain variables $\boldsymbol{\theta}$ to the measurement outcome (in this case the strain as a function of the initiation period and corrosion rate in each of the elements) and $\sigma$ is the error, which can include both a measurement error and model error. It can be seen that Equation (4) is indeed a sum of squares and that the terms $f_{i}$ in Equation (3) are given by $\frac{\left(\bar{y}_{i}-y(\vartheta)\right)}{\sigma}$. When the prior distribution is Gaussian, in a similar way $F_{M A P r}$ can be written according to Equation (5) when disregarding the constant terms:

$$
F_{M A P r}=\sum_{i}^{n} \frac{\left(\vartheta_{i}-\mu\right)^{2}}{\sigma^{2}}
$$

Here, $\theta_{i}$ is the $\mathrm{i}^{\text {th }}$ variable in $\boldsymbol{\theta}$ and has mean $\mu$ and standard deviation $\sigma$. It should be pointed out that Equation (4) and Equation (5) assume that both the measurement results and the variables $\boldsymbol{\theta}$ are mutually independent. When these are correlated, a method of decorrelation can be used that transforms the prediction errors in uncorrelated residuals by making use of the eigenvectors and eigenvalues of the covariance matrix (Simoen, 2013). In the following (i.e., section 5 and further), random fields are used to model the initiation period and corrosion rate in all elements. Hence, the variables $\boldsymbol{\vartheta}$ in the equations given above represent the initiation period $T_{i}$ and the corrosion rate $V_{\text {corr }}$ in the elements in which the structure is discretized. The application of random fields leads to correlated variables along the space over which the random field spans. The uncorrelated terms required in Equation (5) are obtained by applying the Karhunen-Loève decomposition for discretization of the random fields (Vanmarcke, 2010).

An approximate method for the hierarchical Bayesian updating based on (Behmanesh et al., 2015) is used in this work,. For this purpose, conjugate priors are considered. According to (Behmanesh et al., 2015), the prior distributions of the variances are given by an inverse gamma distribution with parameters $\alpha$ and $\beta$. The mean and standard deviation of the inverse gamma distribution are then given by:

$$
\mu=\frac{\beta}{\alpha-1} \text { and } \sigma^{2}=\frac{\beta^{2}}{(\alpha-1)^{2}(\alpha-2)}
$$

For the mean values of the initiation period and corrosion rate, non-informative priors are considered according to (Behmanesh et al., 2015), assuming uniform distributions.

The corrosion parameters are approximated based on an iterative method, making use of the MAP estimates and under the assumption of the mentioned prior distributions. In the following scheme, $\hat{\mu}_{T_{i}}^{j}$ and $\hat{\sigma}_{T_{i}}^{j}$ 
represent the estimate of the mean and variance of the initiation period at step $j$. The iterations consist of the calculation of the error between the mean and standard deviation of the current and previous step and comparing this with the convergence criterion. In a next step, the MAP estimates are generated for the different available datasets, based on which the mean and standard deviation are estimated.

1. First, an initial estimate is made of the parameters of the initiation period and corrosion rate:

$\hat{\mu}_{T_{i}}^{0}, \hat{\mu}_{V_{c o r r}}^{0}, \widehat{\Sigma}_{T_{i}}^{0}$ and $\widehat{\Sigma}_{V_{c o r r}}^{0}$. These initial estimates can be taken equal to the prior mean values. The errors on the estimated parameters for the initiation period are calculated as:

$$
\varepsilon_{\widehat{\mu}_{T_{i}}}=\frac{\hat{\mu}_{T_{i}}^{j}-\hat{\mu}_{T_{i}}^{j-1}}{\hat{\mu}_{T_{i}}^{j-1}} \text { and } \varepsilon_{\widehat{\sigma}_{T_{i}}^{2}}=\frac{\hat{\sigma}_{T_{i}}^{2}-\hat{\sigma}_{T_{i}}^{j-1}}{\hat{\sigma}_{T_{i}}^{2 j-1}}
$$

Similar expressions are used for the errors on the estimated parameters of the corrosion rate.

2. As long as the error on the estimated parameters is larger than 0.01 :

a. Generate a MAP estimate of $T_{i}$ and $V_{\text {corr }}$ for each dataset $t$ (of the $N_{t}$ datasets available) according to Equation (1), i.e. $\widehat{T}_{l_{t}}^{j}$ and ${\widehat{V_{\text {corr }}}}_{t}^{j}$.

b. Generate an estimate of the mean values of the initiation period and corrosion rate based on the calculated MAP estimates:

$$
\begin{gathered}
\hat{\mu}_{T_{i}}^{j}=\frac{1}{N_{t}} \sum_{t=1}^{N_{t}} \widehat{T}_{l_{t}}^{j} \\
\hat{\mu}_{V_{c o r r}}^{j}=\frac{1}{N_{t}} \sum_{t=1}^{N_{t}}{\widehat{V_{c o r r}}}_{t}^{j}
\end{gathered}
$$

c. Generate an estimate of the variance of the initiation period and corrosion rate based on the calculated MAP estimates:

$$
\begin{gathered}
\hat{\sigma}_{T_{i}}^{2}{ }^{j}=\frac{\beta_{T_{i}}+\frac{\sum_{t=1}^{N_{t}}\left(\widehat{T}_{l_{t}}^{j}-\hat{\mu}_{T_{i}}^{j}\right)^{2}}{2}}{\alpha_{T_{i}}+\frac{N_{t}}{2}-1} \\
\hat{\sigma}_{V_{\text {corr }}}^{2}{ }^{j}=\frac{\beta_{V_{\text {corr }}}+\frac{\sum_{t=1}^{N_{t}}\left({\widehat{V_{\text {corr }}}}^{j}-\hat{\mu}_{V_{\text {corr }}}^{j}\right)^{2}}{2}}{\alpha_{V_{\text {corr }}}+\frac{N_{t}}{2}-1}
\end{gathered}
$$

The posterior distributions of the means are then given by (Behmanesh et al., 2015):

$$
\begin{gathered}
f^{\prime \prime}\left(\mu_{T_{i}} \mid .\right)=N\left(\hat{\mu}_{T_{i}}, \frac{1}{N_{t}} \hat{\sigma}_{T_{i}}\right) \\
f^{\prime \prime}\left(\mu_{V_{c o r r}} \mid .\right)=N\left(\hat{\mu}_{V_{c o r r}}, \frac{1}{N_{t}} \hat{\sigma}_{V_{c o r r}}\right)
\end{gathered}
$$

This is based on the assumption that the posterior distribution approaches a normal distribution for a sufficiently large dataset. The posterior distributions of the variances are then given by (Behmanesh et al., 2015):

$$
\begin{gathered}
f^{\prime \prime}\left(\sigma_{T_{i}}^{2} \mid .\right)=\text { InverseGamma }\left(\left(\frac{N_{t}}{2}+\alpha_{T_{i}}\right), \beta_{T_{i}}+\frac{1}{2} \sum_{t=1}^{N_{t}}\left(\widehat{T}_{i_{t}}-\hat{\mu}_{T_{i}}\right)^{2}\right) \\
f^{\prime \prime}\left(\sigma_{V_{\text {corr }}}^{2} \mid .\right)=\text { InverseGamma }\left(\left(\frac{N_{t}}{2}+\alpha_{V_{\text {corr }}}\right), \beta_{V_{\text {corr }}}+\frac{1}{2} \sum_{t=1}^{N_{t}}\left(\widehat{V}_{\text {corr }_{t}}-\hat{\mu}_{V_{\text {corr }}}\right)^{2}\right)
\end{gathered}
$$

In (Behmanesh et al., 2015) stiffness distributions are updated based on available vibration data. The main objective of that work was to introduce a probabilistic FE model updating technique for identification of civil structural systems under changing ambient or environmental conditions, enabling probabilistic damage identification of structural systems. However, the purpose was not to update the parameters of the underlying degradation phenomenon, which would enable predictions of the remaining service life of the structure. Moreover, the distributions for the stiffness are uncorrelated in (Behmanesh et al., 2015). In this work, the work of (Behmanesh et al., 2015) is used as a basis to update the random fields of corrosion variables influencing the stiffness of the structure (see section 3). The influence of different assumptions on the modelling of the random fields is investigated by looking at the resulting distribution of the reliability index. 
In this work, the posterior distributions are derived based on strain measurements in a similar way as explained in (Vereecken, Botte, Lombaert, \& Caspeele, 2021), but now using MAP estimates instead of MCMC (Markov Chain Monte Carlo) sampling, since in the current work the distributions of the parameters of the initiation period and corrosion rate are updated and not the distributions of the variables itself. The relation between the strains along the structure and the corrosion variables is obtained by a finite element model. However, to save computational time, a response surface is fit to these relations, which is then used in the generation of the MAP estimates. The response surface is a polynomial response surface of third degree (Bucher, 2009), which is a function of the corrosion degree in all elements in which the structure is subdivided. The response surface has been generated for different orders, where the fit of each of the response surfaces has been evaluated by calculating the coefficient of determination $R^{2}$, taking into account a correction for the number of unknowns in the response surface when considering higher orders. When evaluating the influence of the corrosion degree on the strains, the behaviour is non-linear; hence, a response surface of degree 1 does not seem appropriate. When comparing a response surface of degree 2 and degree 3 , the coefficient of determination $R^{2}$ (with a correction for the number of unknowns) is much larger and a better fit is found for the latter.

In (Vereecken et al., 2021) a correlation model for the random field is selected among different candidate models without investigating the influence of this assumption. Moreover, the distributions of the variables describing the corrosion process are updated directly instead of the distributions of their parameters (i.e. the distributions of e.g. their mean and standard deviation). The focus of the previously mentioned work is on incorporating information from static strain data and modal data in the assessment of corrosion and how the incorporation of different data types influences the posterior distribution of the corrosion degree. Hence, it is more important to arrive at an accurate representation of the latter and MCMC is more recommended than an approximation method. In the present work, the posterior distribution of the corrosion variables should be derived for different values of the parameters (i.e. mean and standard deviation), inducing a large computational burden. Therefore, a more computationally efficient procedure is applied based on MAP estimates. After convergence, the MAP estimates of all the updating parameters provide the global maxima of the posterior joint distribution, approximating the latter. Since in the present work, the focus is on the impact of the choice of the correlation model of the random field representing the corrosion process on the posterior distribution of the reliability index, the use of the MAP estimates can be justified. It limits the computational time a lot, enabling the comparison of multiple correlation models. Furthermore, the minor approximation error made by the use of MAP instead of MCMC will not induce large differences in reliability index.

\section{CORRELATION MODELS FOR RANDOM FIELDS APPLIED TO CORROSION VARIABLES}

To model the spatial variation of concrete properties or corrosion, random fields can be used. When discretized, the Gaussian random field is defined by a mean vector of size $n \times 1$ and a covariance matrix of size $n \times n$, with $n$ the number of points at which the random field is evaluated. Random fields with a different marginal distribution can be generated assuming an underlying Gaussian random field and applying a Nataf transformation (Liu \& Der Kiureghian, 1986). The off-diagonal elements in the covariance matrix of the random field are a function of the correlation $\rho$ between two points at which the random field is evaluated. In literature, different assumptions on the correlation models for corrosion variables can be found. Criel, Caspeele, \& Taerwe (2014) summarize some correlation lengths commonly found in literature for corrosion and concrete variables, as presented in Table 1.

Table 1 Correlation lengths as summarized in (Criel et al., 2014)

\begin{tabular}{c|c|c}
\hline Variable & Correlation lengths & References \\
\hline Concrete cover $c$ & $1 \mathrm{~m}-2 \mathrm{~m}-3.5 \mathrm{~m}$ & $\begin{array}{c}\text { (Li, Vrouwenvelder, Wijnants, \& Walraven, 2004; Stewart } \\
\text { \& Mullard, 2007; Straub, 2011) }\end{array}$ \\
$\begin{array}{c}\text { Surface chloride } \\
\text { concentration } C_{s}\end{array}$ & $1 \mathrm{~m}-1.96 \mathrm{~m}-2 \mathrm{~m}$ & (Duprat, 2007; Engelund, 1997; Straub, 2011; Vu, 2003) \\
$\begin{array}{c}\text { Concrete compressive } \\
\text { strength } f_{c}\end{array}$ & $3.5 \mathrm{~m}$ & (Duprat, 2007) \\
\hline
\end{tabular}

The correlation models for the correlation coefficient $\rho$ mentioned in (Criel et al., 2014) are the exponential correlation model (Equation (9)), the squared exponential model (Equation (10)) and the Matérn model (Equation (11)). The last one depends not only on the correlation length $l_{c}$, but also on a smoothness parameter $v$. In Equations (9) to (11), $\Delta$ is the distance between two points in the random field, and $l_{c}$ the correlation length. In Equation (11), $\Gamma($.$) is the Gamma function, K_{v}($.$) the \operatorname{BesselK}(v,$.$) function and v$ a smoothness parameter. 


$$
\begin{gathered}
\rho=\exp \left(-\frac{\Delta}{l_{c}}\right) \\
\rho=\exp \left(-\frac{\Delta^{2}}{l_{c}^{2}}\right) \\
\rho=\frac{1}{\Gamma(v) 2^{v-1}}\left(\frac{2 \sqrt{v} \Delta}{\theta}\right)^{v} K_{v}\left(\frac{2 \sqrt{v} \Delta}{\theta}\right) \text { with } \theta=l_{c} \frac{\sqrt{\pi} \Gamma\left(v+\frac{1}{2}\right)}{\sqrt{v} \Gamma(v)}
\end{gathered}
$$

The importance of the correlation model and correlation length depends on the quantity of interest and the investigated case. The degree of spatial correlation between two values of the random field depends on both the correlation model and the correlation length. Although in general the correlation will decrease as the distance becomes larger relative to the correlation length, the actual degree of correlation also depends on the model, so both cannot be considered independently when discussing their importance for a specific quantity of interest.

In (Straub, 2011), the exponential correlation model is applied, with the correlation lengths mentioned in Table 2. In (Tran et al., 2012), a correlation length of $1.1 \mathrm{~m}$ is considered for the diffusion coefficient and Vu \& Stewart (2005) assume a squared exponential correlation model with lengths as given in Table 3. In (Straub, Malioka, \& Faber, 2009), an exponential correlation model is considered with a correlation length of $80 \mathrm{~cm}$ for the chloride conductivity. In (Hajializadeh et al., 2015), a squared exponential correlation is considered with a correlation length of $1 \mathrm{~m}$ for the concrete cover and concrete compressive strength and in (Stewart \& Mullard, 2007) also a squared exponential correlation model is considered, but with correlation length $2 \mathrm{~m}$ for concrete cover, concrete compressive strength and surface chloride concentration. For the surface chloride concentration and concrete cover, Ying, Vrouwenvelder, \& Wijnants (2004) assume a squared exponential correlation model with a lower bound $\rho_{0}$ of 0.5 or 0 and a correlation length of $2 \mathrm{~m}$ for the concrete cover and surface chloride concentration, according to Equation (12). Furthermore, also (Stewart \& Suo, 2009) assume a correlation length of $2 \mathrm{~m}$ combined with an exponential correlation function for concrete cover, concrete compressive strength and surface chloride concentration:

$$
\rho=\rho_{0}+\left(1-\rho_{0}\right) \cdot \exp \left(-\frac{\Delta^{2}}{l_{c}^{2}}\right)
$$

Table 2 Correlation lengths as summarized in (Straub, 2011)

\begin{tabular}{l|l}
\hline Variable & Correlation lengths \\
\hline Concrete cover $c$ & $1 \mathrm{~m}$ \\
Surface chloride concentration $C_{s}$ & $2 \mathrm{~m}$ \\
Critical chloride concentration $C_{c r}$ & $2 \mathrm{~m}$ \\
Diffusion coefficient of concrete $D$ & $2 \mathrm{~m}$ \\
\hline
\end{tabular}

Table 3 Correlation lengths as summarized in (Vu \& Stewart, 2005)

\begin{tabular}{l|l}
\hline Variable & Correlation lengths \\
\hline Concrete cover $c$ & $2 \mathrm{~m}$ \\
Surface chloride concentration $C_{s}$ & $1.96 \mathrm{~m}-1 \mathrm{~m}-2 \mathrm{~m}$ \\
Water/cement ratio $w / c$ & $0.5 \mathrm{~m}$ \\
Young's modulus of the concrete $E_{c}$ & $2 \mathrm{~m}$ \\
Poisson coefficient of concrete $v$ & $2 \mathrm{~m}$ \\
Concrete compressive strength $f_{c}$ & $2 \mathrm{~m}$ \\
\hline
\end{tabular}

\section{ILLUSTRATIVE EXAMPLE USED FOR THE QUANTITATIVE COMPARISON}

In this work, a simply supported beam subjected to chloride-induced corrosion is considered. The beam has a length of $4 \mathrm{~m}$, a height of $500 \mathrm{~mm}$ and a width of $300 \mathrm{~mm}$. The initial reinforcement section equals $942.5 \mathrm{~mm}^{2}$ (i.e. three bars of $20 \mathrm{~mm}$ diameter). To account for spatial variation of the corrosion process, the beam is subdivided in eight elements of $0.5 \mathrm{~m}$. The reinforcement area of the beam $A_{s}$ varies in time according to Equation (13) (Duracrete, 2000; Lay, Schieß1, \& Cairns, 2003; Stewart \& Rosowsky, 1998) since the beam is considered to be subjected to chloride-induced corrosion. The prior distributions of the corrosion parameters used in this example are given in Table 4 . These are based on a CEMI/42.5 with a water/cement ratio of 0.45 and a cyclic wet-dry exposure class. The distribution of the initiation period is based on the distributions of the parameters appearing in Equation (14) (fib, 2006). To account for spatial variation, the initiation period and corrosion rate are modelled by random fields. 


$$
\begin{gathered}
\alpha(t)=\frac{A_{s, 0}-A_{s}(t)}{A_{s, 0}} \text { with } A_{s}(t)=\left(r_{0}-x(t)\right)^{2} \cdot \pi \cdot n_{r} \\
\begin{array}{c}
\text { and } x(t)=V_{c o r r} \cdot \alpha_{p} \cdot\left(t-T_{i}\right) \\
T_{i}=\frac{1}{4 D} \frac{c^{2}}{\left(\operatorname{erf}^{-1}\left(1-\frac{C_{c r}}{C_{s}}\right)\right)^{2}}
\end{array}
\end{gathered}
$$

In Equation (13), $\alpha(t)$ is the corrosion degree at time $t, A_{s, 0}$ the initial reinforcement area, $A_{s}(t)$ the reinforcement area at time $t, r_{0}$ the initial reinforcement radius, $x(t)$ the reduction in reinforcement radius due to corrosion and $n_{r}$ the number of reinforcement bars. For the other variables in Equation (13) and Equation (14) reference is made

\begin{tabular}{|c|c|c|c|c|c|}
\hline Variable & $\begin{array}{l}\text { Symbol } \\
\text { [unit] }\end{array}$ & Mean & $\begin{array}{l}\text { Standard } \\
\text { deviation }\end{array}$ & Distribution & Reference \\
\hline \multicolumn{6}{|l|}{ Propagation } \\
\hline Pitting factor & $\alpha_{p}[-]$ & 2 & - & Deterministic & (Duracrete, 2000) \\
\hline $\begin{array}{l}\text { Mean corrosion rate while } \\
\text { corrosion is active }\end{array}$ & $\begin{array}{l}V_{\text {corr, } a} \\
{[\mathrm{~mm} / \mathrm{yr} .]}\end{array}$ & 0.03 & 0.04 & Weibull & (Lay et al., 2003) \\
\hline Time of wetness & ToW [-] & 0.75 & 0.2 & Normal & (Lay et al., 2003) \\
\hline \multicolumn{6}{|l|}{ Initiation } \\
\hline Initiation period & $T_{i}[$ years] & 22 & 10 & Lognormal & $\begin{array}{l}\text { (Vereecken et al., } \\
\text { 2021) }\end{array}$ \\
\hline $\begin{array}{l}\text { Diffusion coefficient of } \\
\text { concrete }^{*}\end{array}$ & $D\left[m m^{2} / y r.\right]$ & 20 & 10 & Lognormal & (fib, 2006) \\
\hline $\begin{array}{l}\text { Surface chloride } \\
\text { concentration }\end{array}$ & $\begin{array}{l}C_{s}[w t .- \\
\% / c]\end{array}$ & 2 & 0.9 & Lognormal & (fib, 2006) \\
\hline $\begin{array}{l}\text { Critical chloride } \\
\text { concentration }^{*}\end{array}$ & $\begin{array}{l}C_{c r}[w t .- \\
\% / c]\end{array}$ & 0.6 & 0.15 & Lognormal & (fib, 2006) \\
\hline Concrete cover ${ }^{*}$ & $c[\mathrm{~mm}]$ & 30 & 5 & Lognormal & (fib, 2006) \\
\hline
\end{tabular}
to Table 4.

Table 4 Distributions of variables related to the corrosion model

${ }^{*}$ These distributions are used for the determination of the parameters of $T_{i}$ applying a Taylor approximation

The failure probability of the beam is evaluated based on the (simplified) limit state equation for failure due to bending given by (CEN, 2005):

$$
g(\boldsymbol{X}, t)=K_{R} A_{s}(x, t) f_{y}\left(h-c-0.5 \frac{A_{s}(x, t) f_{y}}{b f_{c}}\right)-K_{E}(G+Q)
$$

The variables used in Equation (15) and the corresponding distributions are given in Table 5. Here a concrete class C30/37 and reinforcement steel BE500 are considered. For the variable load effect, the distribution for a 1-year reference period is used to calculate the annual probability of failure and the characteristic value of the variable load equals $30 \mathrm{kN}$ (point load at midspan).

Table 5 Distributions to evaluate the bending limit state of the beam (JCSS, 2001)

\begin{tabular}{llllll}
\hline Variable & Symbol & Units & Mean & $\begin{array}{l}\text { Standard } \\
\text { deviation }\end{array}$ & Distribution \\
\hline Resistance model uncertainty & $K_{R}$ & - & 1 & 0.05 & Lognormal \\
\hline Reinforcement yield stress & $f_{y}$ & $\mathrm{MPa}$ & 550 & 11 & Lognormal \\
\hline Concrete compressive strength & $f_{c}$ & $\mathrm{MPa}$ & 38.8 & 4.6 & Lognormal \\
\hline $\begin{array}{l}\text { Load model uncertainty } \\
\text { Permanent load effect }\end{array}$ & $K_{E}$ & - & 1 & 0.10 & Lognormal \\
\hline $\begin{array}{l}\text { Variable load effect (1year reference } \\
\text { period) }\end{array}$ & $Q$ & $\mathrm{kNm}$ & 50 & 2.5 & Normal \\
\hline \begin{tabular}{l} 
Concrete cover \\
\hline
\end{tabular} & $c$ & $\mathrm{kNm}$ & 6 & 6.6 & Gumbel \\
\hline
\end{tabular}


In the following, the parameters of the distributions of the initiation period and corrosion rate are modelled as uncertain themselves. This will lead to a distribution of the reliability index. The latter distribution is calculated by Latin Hypercube Sampling (LHS) of the parameters of the initiation period and corrosion rate. One hundred Latin Hypercube samples are generated. For each of these samples (i.e., sampled values for the mean and standard deviation of initiation period and corrosion rate), the reliability index is calculated. This calculation of the reliability index is based on the assumption of a series system, where first the reliability index is calculated independently for each of the eight elements in the discretization of the beam. Next, the probability of failure is calculated based on these individual reliabilities and additionally considering their mutual correlation, estimated based on the sensitivity factors resulting from the FORM analysis and the correlation between the beam elements resulting from the random field modelling of the initiation period and corrosion rate. This approach is also described in (Vereecken, Botte, Lombaert, \& Caspeele, 2020).

In literature, very little information is available on the distributions for mean and standard deviation of corrosion variables. Hence, vague prior distributions are considered. For the initiation period, the mean of the standard deviation is taken equal to 10 years, which is based on the standard deviation provided in Table 4 . The standard deviation of the standard deviation is taken equal to 3.5 years based on the assumptions made in (Botte, 2017). Based on the same reference, for the corrosion rate the mean of the standard deviation is taken equal to 0.02 $\mathrm{mm} /$ year based on Table 4 and the standard deviation of the standard deviation is taken equal to $0.007 \mathrm{~mm} / \mathrm{year}$. The parameters of the Gamma distributions applied to model the variance of initiation period and corrosion rate are $\alpha=2.5$ and $\beta=2.5$ for the initiation period and $\alpha=150$ and $\beta=6 \mathrm{e}-4$ for the corrosion rate. For the means of the initiation period and corrosion rate, non-informative priors are considered according to (Behmanesh et al., 2015). For the mean of the initiation period, a uniform prior between 0 and 40 years is considered and for the mean of the corrosion rate a uniform prior between 0 and $0.07 \mathrm{~mm} / \mathrm{year}$. Hence, it is assumed a priori that the initiation period will not exceed 40 years and the corrosion rate will not be larger than $0.07 \mathrm{~mm} / \mathrm{year}$.

\section{INFLUENCE OF THE CORRELATION MODEL ON THE POSTERIOR DISTRIBUTION OF THE RELIABILITY INDEX}

Based on the literature survey provided in section 3, different correlation models are investigated. These are designated according to Table 6 . The different correlation models are also illustrated in Figure 1. In this work, the correlation model will be taken the same for both the initiation period and corrosion rate. The seven correlation models chosen by the authors are the ones most commonly appearing in literature in this domain of application (i.e. concrete structures), with correlation lengths that are regularly found in the same literature. Also, since the different correlation models differ close to the origin, the correlation between an element and the neighbouring element will differ. The (asymptotic) behaviour with growing distance from the origin will define a different correlation between elements located further away from each other. It is difficult to obtain a good idea on the behaviour near the origin based on experimental data. This results from the fact that the samples should then be located very close to each other, which often proves to be physically difficult or impossible. This results in different assumptions on amongst others the correlation model. Moreover, a lack of experimental datasets to estimate the correlation models and correlation lengths results in the use of different models and varying correlation lengths in literature. In Figure 1, it can be seen that some of the correlation models asymptotically reach a zero value with increasing distance, while some models reach a finite nonzero value of the correlation, regardless of how large the distance is, or reach this asymptotic behaviour only at larger distances.

Table 6 Considered correlation models

\begin{tabular}{c|c|c}
\hline Model number & Correlation model & Parameter values \\
\hline 1 & Equation (10) & $l_{c}=1 \mathrm{~m}$ \\
2 & Equation (10) & $l_{c}=2 \mathrm{~m}$ \\
3 & Equation (9) & $l_{c}=1 \mathrm{~m}$ \\
4 & Equation (9) & $l_{c}=2 \mathrm{~m}$ \\
5 & Equation (9) & $l_{c}=3.5 \mathrm{~m}$ \\
6 & Equation (12) & $l_{c}=2 \mathrm{~m}, \rho_{0}=0.5$ \\
7 & Equation (11) & $v=1, l_{c}=1 \mathrm{~m}$ \\
\hline
\end{tabular}




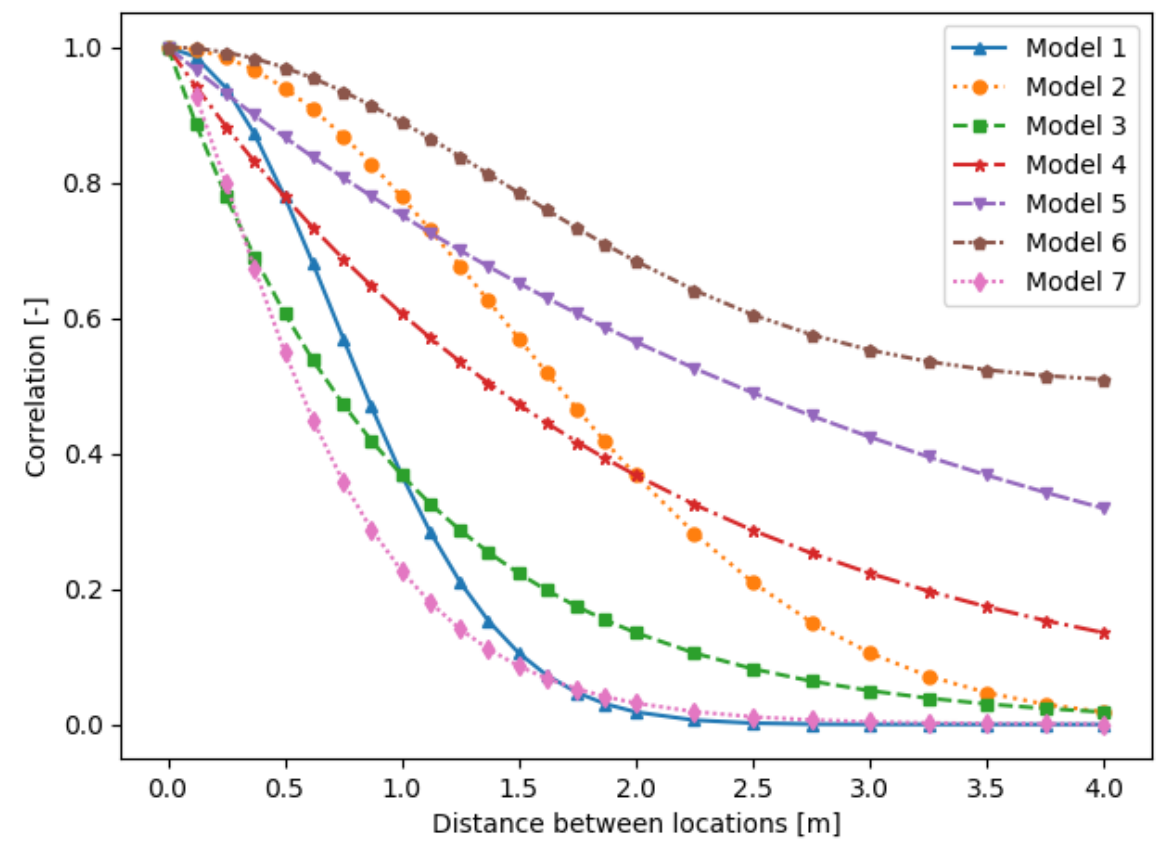

Figure 1 Graphical representation of the different correlation models as summarized in Table 6

To investigate the influence of the correlation model, measurement results (in this work static strain data) are generated for each of these correlation models. Then, for each of these measurement results, the posterior distributions of the parameters of the initiation period and corrosion rate are estimated. Based on the posterior distributions of the parameters of the initiation period and corrosion rate, the distribution of the reliability index is calculated. This posterior distribution of the reliability index is then compared with the one derived based on the actual correlation model used to generate the measurement result. As such, it will be investigated whether one of the correlation models has a better performance than the others, independent of the actual correlation model. As such, the influence of the modelling error of applying a correlation model not corresponding to the model actually fitting the data is investigated. In the end, the most 'robust' model is searched, i.e. the model leading to the smallest error when there is a mismatch between the model that is used to generate the data and the model that is used in the updating procedure (further referred to as calibration). It should be pointed out that the modelling error due to an inappropriate choice of the random field is only one of the possible sources of modelling errors that can be encountered in real applications.

The measurement results (i.e. static strain data) are generated based on a finite element model of the beam as described in (Vereecken et al., 2021), and this for the different correlation models. The load applied to the beam consists of a point load of $30 \mathrm{kN}$ at midspan. The measured strains are in the order of magnitude of -65 to $55 \mu \varepsilon$. The reinforcement area is calculated based on a sample of the random fields for the initiation period and corrosion rate. To these finite element results, a sample of a measurement error $(\mathrm{N}(0,0.5 \mu \varepsilon))$ is added. The updating of the mean values and standard deviations of the initiation period and corrosion rate is performed according to section 2. These posterior distributions are derived seven times, i.e. each time the prior distribution is based on one of the seven correlation models as given in Table 6 . The influence on the posterior distribution when assuming different priors is most clear for the mean value of the mean of the initiation period and corrosion rate. When the underlying correlation model corresponds for instance to model 1 , the posterior mean value of the mean of the initiation period is illustrated in Figure 2. Similarly, the posterior mean value of the mean of the corrosion rate is illustrated in Figure 3. These figures illustrate that the posterior mean values of the initiation period and corrosion rate will differ for different prior assumptions on the correlation model of the random fields. 


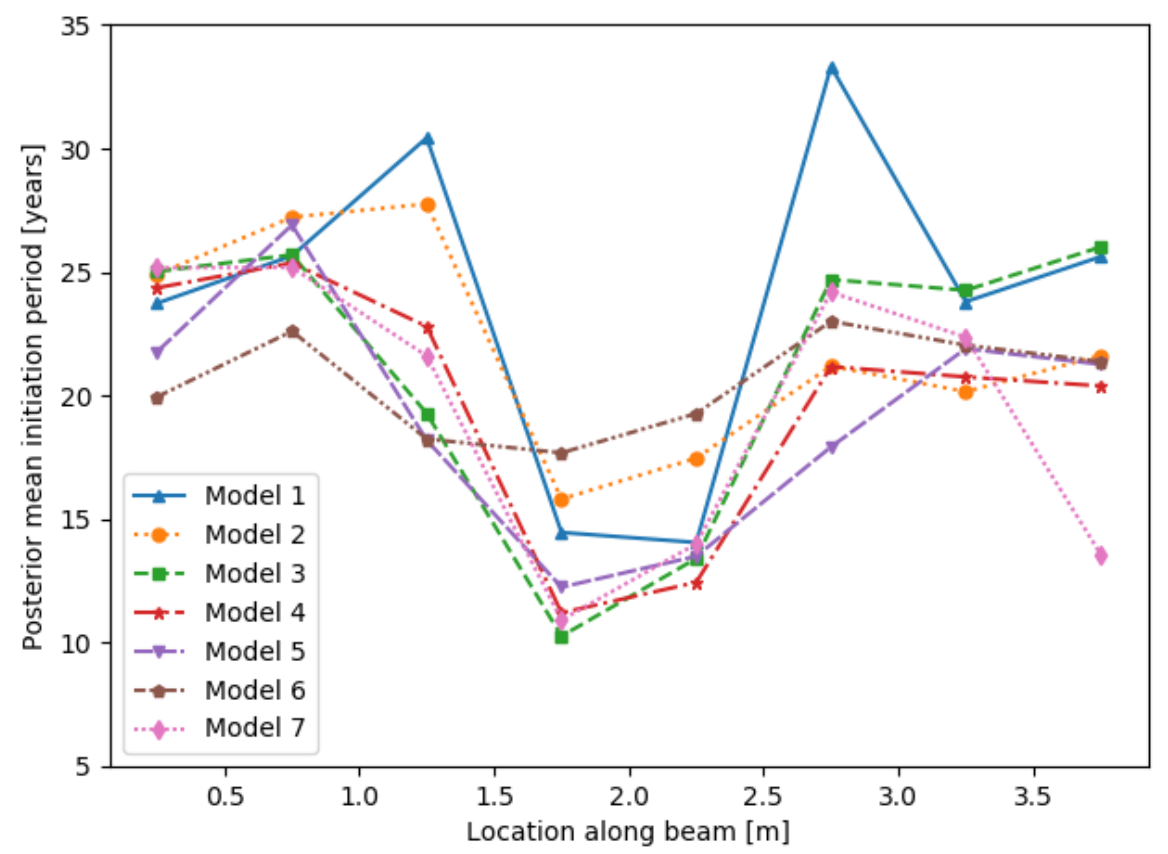

Figure 2 Posterior mean value of the mean of the initiation period for the different correlation models when the underlying correlation model is model 1

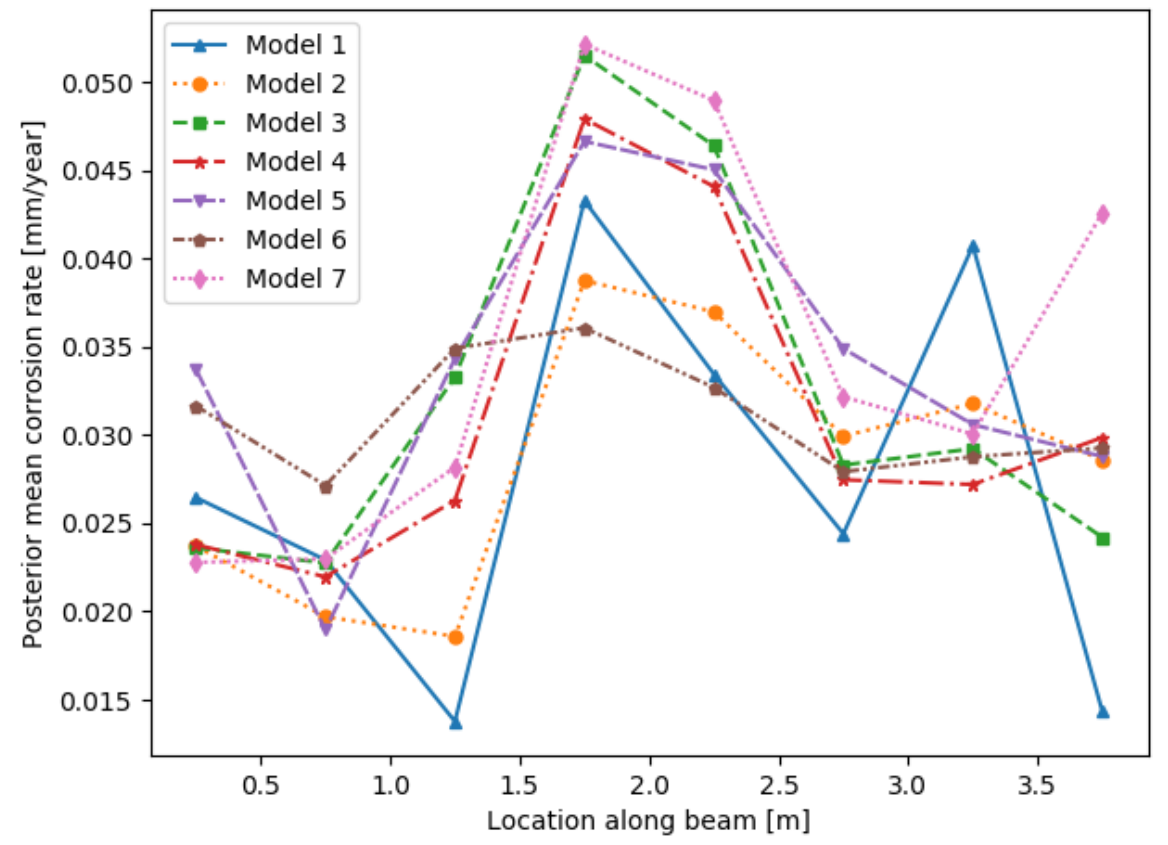

Figure 3 Posterior mean value of the mean of the corrosion rate for the different correlation models when the underlying correlation model is model 1

Based on the posterior distributions of the parameters of the initiation period and corrosion rate, the corresponding reliability indices are estimated and shown in Figure 4. These posterior reliability indices are given relative to the posterior mean reliability index when updating is performed based on the actual correlation model. It can be seen that the mismatch between the prior correlation model in the calibration and the actual correlation model results in different values for the posterior reliability index. The models given as headings of the different subfigures in Figure 4 refer to the actual underlying correlation model.

A correlation model is a robust choice if the resulting posterior distribution of the reliability index based on this prior model approximates the actual posterior distribution (which follows from the actual underlying model). Hence, when model 1 is considered as the prior model, it can be seen that when the actual correlation model is model 2 (Figure 4 (b)), the dotted line (representing the posterior mean of the reliability index) lies within 
the interquartile range (IQR) of the boxplot of model 1. The same holds for model 3 (Figure 4 (c)). Hence, these models are summarized in Table 7 in the column 'Models in IQR' for the prior model 1 . When model 4 is the actual model (Figure 4 (d)), the dotted line crosses the lines between minimum and maximum of the boxplot (the so-called whiskers). The same again holds for actual models 5 and 6 (Figure 4 (e) and (f)). Hence, in Table 7, for the prior model 1, models 4, 5 and 6 are summarized in the column 'Models in whiskers'.

A similar analysis can be done for the other six prior models. These results are also summarized in Table 7. Here it can be seen that when model 2 or model 6 is used as a prior, the actual mean reliability index related to the other (actual) models is always inside the boxplot for model 2 or model 6 (i.e. within the IQR or within the whiskers). For both prior models, none of the other models is mentioned in the column 'Models outside of the boxplot'. When comparing model 2 with model 6 , model 2 performs better since here the number of actual models for which the mean of the reliability index lies within the interquartile range of the boxplot for prior model 2 is larger (i.e. more models are mentioned in the column 'Models in IQR'). For each model, the deviation between the mean reliability index and the mean reliability index of the actual model is also calculated. The average of these deviations is given in the column ' $\left(\Delta \beta / \beta_{\text {act }}\right)_{\text {average' }}$ and the maximum of these deviations is given in the column ' $\left(\Delta \beta / \beta_{\text {act }}\right)_{\max }$ '. 


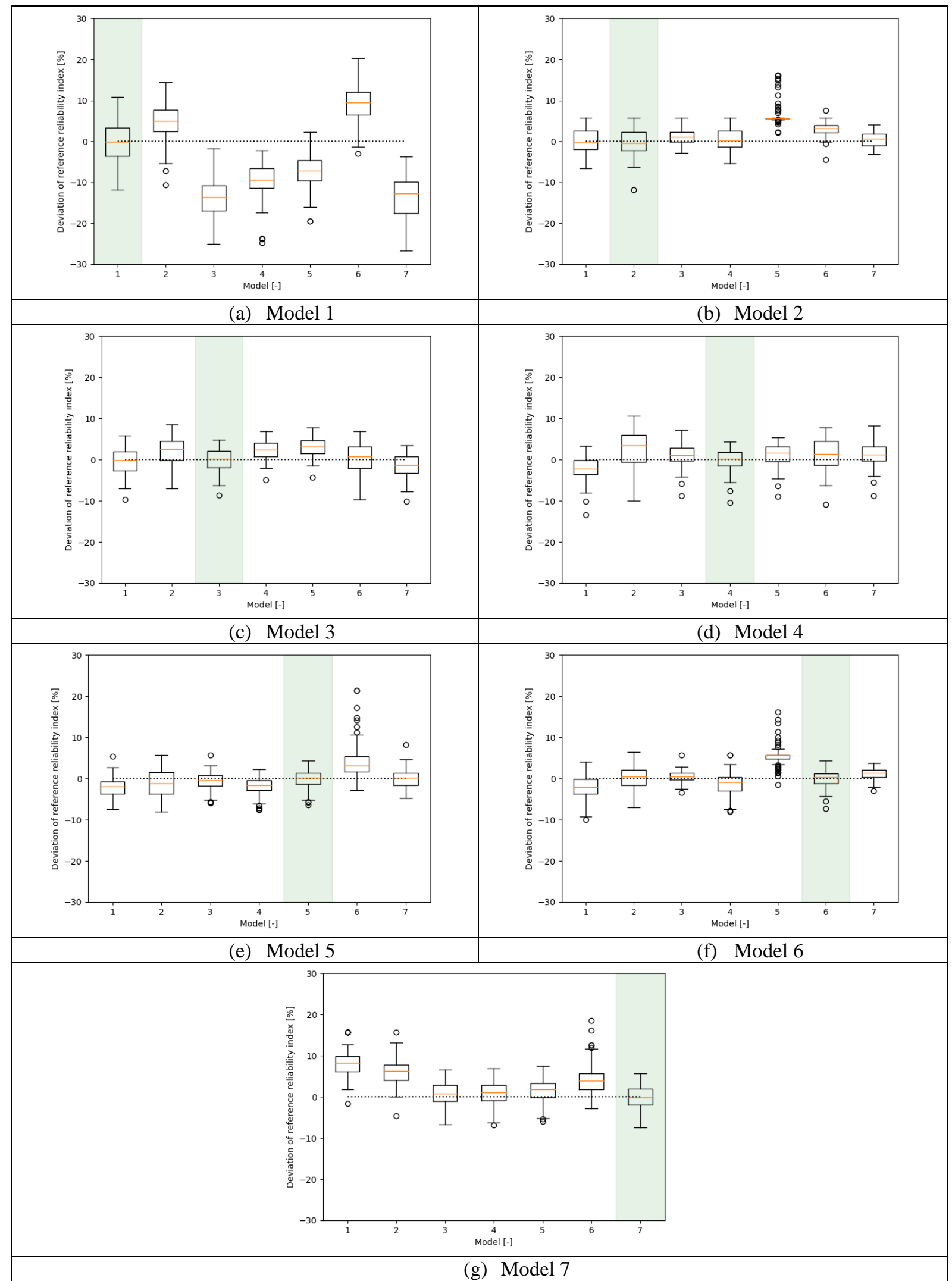

Figure 4 Posterior distributions of the reliability indices for the different actual correlation models (indicated by the captions of the subplots). The dotted line in each graph indicates the mean reliability index resulting from the actual correlation model. The vertical axis represents the deviation from the reference reliability index and the horizontal axis represents the model numbers. The shaded area indicates the boxplot of the actual correlation model. 
Table 7 Comparison of the different models based on observations from Figure 4

\begin{tabular}{cccccc}
\hline $\begin{array}{c}\text { Prior } \\
\text { correlation } \\
\text { model }\end{array}$ & $\begin{array}{c}\text { Models with } \\
\text { mean reliability } \\
\text { index in IQR }\end{array}$ & $\begin{array}{c}\text { Models with } \\
\text { mean reliability } \\
\text { index in } \\
\text { whiskers }\end{array}$ & $\begin{array}{c}\text { Models with mean } \\
\text { reliability index } \\
\text { outside of the } \\
\text { boxplot }\end{array}$ & $\begin{array}{c}\left(\boldsymbol{\Delta} / \boldsymbol{\beta}_{\text {act }}\right)_{\text {average }} \\
{[\boldsymbol{\%}]}\end{array}$ & $\begin{array}{c}\left(\boldsymbol{\Delta} \boldsymbol{\beta} / \boldsymbol{\beta}_{\text {act }}\right) \max \\
{[\%]}\end{array}$ \\
\hline 1 & $1,2,3$ & $4,5,6$ & 7 & 2.22 & 8.26 \\
2 & $2,3,4,5,6$ & 1,7 & N/A & 2.45 & 5.99 \\
3 & $2,3,4,5,6,7$ & N/A & 1 & 2.54 & 13.63 \\
4 & $2,4,6,7$ & 3,5 & 1 & 2.37 & 9.37 \\
5 & $4,5,7$ & 1,3 & 2,6 & 3.59 & 7.16 \\
6 & $3,4,6$ & $1,2,5,7$ & N/A & 3.20 & 9.02 \\
7 & $2,3,4,5,7$ & 6 & 1 & 2.58 & 13.71 \\
\hline
\end{tabular}

$* \mathrm{~N} / \mathrm{A}=$ Not Applicable

From the results provided above, it can be concluded that the a priori assumed correlation model may have a large impact on the posterior reliability index. Furthermore, the error on the resulting posterior distribution changes depending on the actual correlation model. The best performing model, i.e. the most robust one, is chosen to be model 2, based on the explanation given above. The mismatch between the reliability index based on calibration with model 2 and the actual distribution of the reliability index is the smallest for this model. In all cases, the actual mean reliability index either lies within the interquartile range (four out of the six cases) or in the interval spanned by the minimum and maximum (no outliers) (two out of the six cases). Also, when the actual correlation model is model 2 , almost all other models provided similar posterior distributions of the reliability index.

When looking at different models with the same correlation function, but a different correlation length, some observations can be made. Models 1 and 2 both have a squared exponential correlation model. When the actual correlation model is model 1 , the second best prior model (after the actual one) is model 2 . The same holds when models 1 and 2 are switched. Model 6 also has a squared exponential correlation function, but with a lower limit on the correlation coefficient. This lower limit leads to a less good approximation of the distribution of the reliability index when the actual model is model 1 or model 2. Models 3, 4 and 5 all have an exponential correlation function. The distribution of the reliability index following from actual model 4 is best approximated when model 3 is used as prior. The approximation is less good for the prior model 5 , due to the larger correlation length. The best approximation (lowest difference in mean reliability index) of model 3, is not given by prior models 4 or 5 , since these reach a zero value more slowly for increasing distances. In a similar way, model 5 is not necessarily better approximated by models of the same 'family' (i.e. an exponential correlation function). Hence, instead of being of the same 'family', the asymptotic behaviour seems more important. In Figure 1, models 1, 2, 3 and 7 all have a zero asymptotic value. Indeed, the best approximation of model 1 is obtained by model 2 , the best approximation of model 2 by models 1 and 7, the best approximation of model 3 by model 1 and the best approximation of model 7 by model 3 .

\section{CASE STUDY}

To investigate whether similar conclusions can be derived for a more extensive and more realistic bridge structure, a case study of an RC girder bridge located in Flanders is considered. The bridge consists of two parts divided by a joint, which are symmetrical with respect to each other (Figure 5). The longitudinal girders of the first part are visualized in Figure 6. The reinforcement of these girders (except for the outer ones and for the small girders next to the joint), is given in Figure 7 (b) and consists of 10 bars (in two layers of five bars) with diameter $40 \mathrm{~mm}$. The reinforcement of the outer girder is given in Figure 7 (a) and of the small girder next to the joint in Figure 7 (c). These girders are reinforced with respectively eight bars of diameter $40 \mathrm{~mm}$ (two layers of four bars) and four bars of diameter $14 \mathrm{~mm}$. The shear reinforcement has a spacing of $200 \mathrm{~mm}$ and consists of stirrups with a diameter of $10 \mathrm{~mm}$. The reinforcement of the deck consists of bars of $14 \mathrm{~mm}$ in two layers with a spacing of $200 \mathrm{~mm}$. 


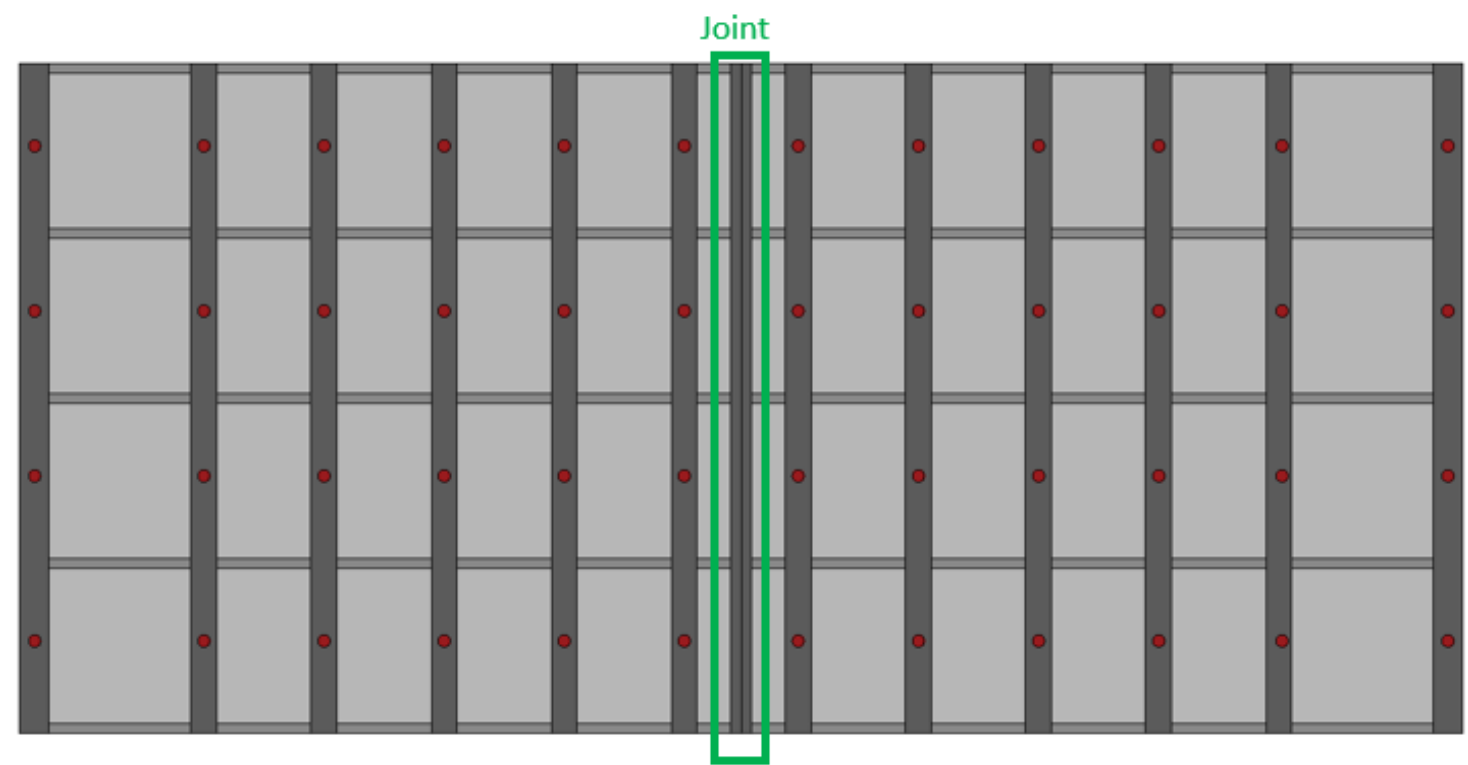

Figure 5 Bottom view of the case study bridge. Measurement locations are indicated with red circles

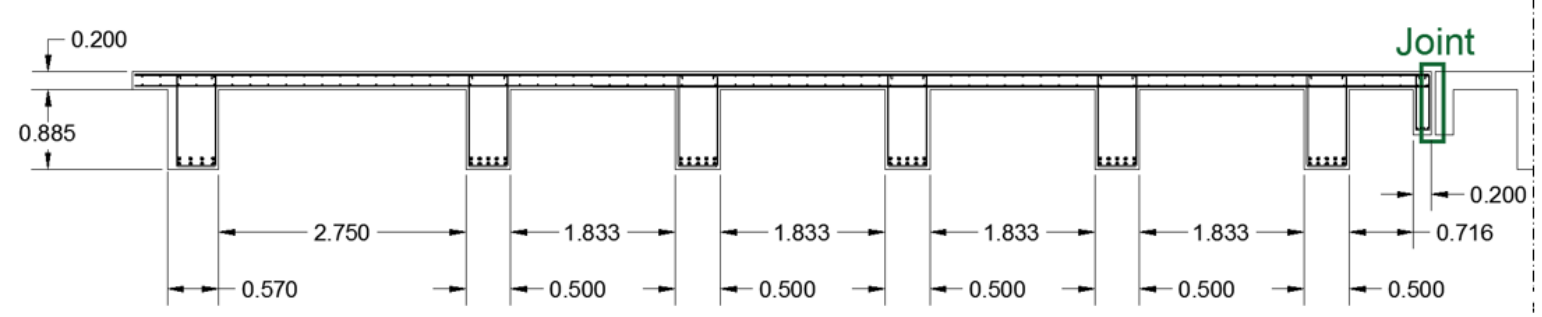

Figure 6 Cross-section of left part of the bridge

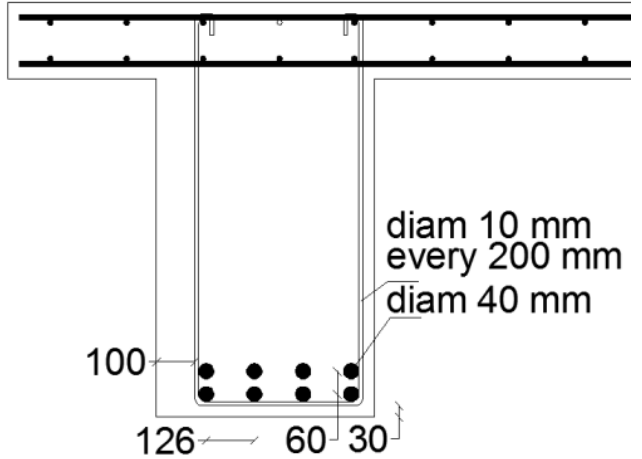

a) Outer girder

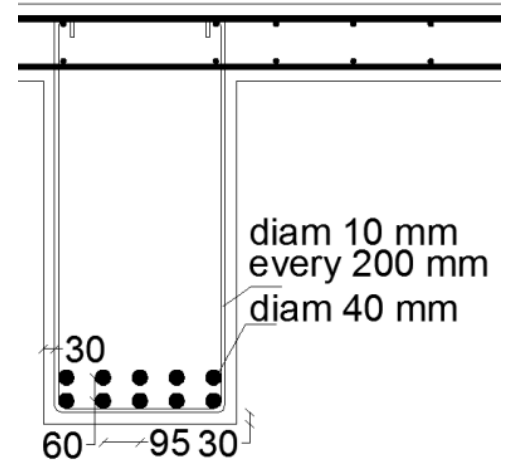

b) Main girders

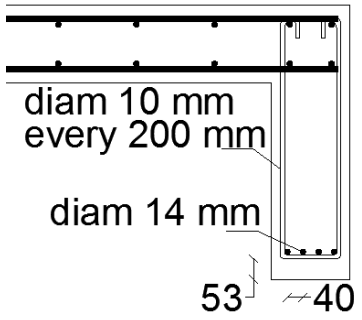

c) Girder at joint

Figure 7 Reinforcement layout of the longitudinal girders

By investigation of the finite element model, the limit states were verified and bending and shear limit states of the girders were most critical. Corrosion was initially considered in the longitudinal girders, transverse stiffeners and in the slab. However, corrosion of the transverse stiffeners and of the slab did not influence the strains to a measurable extent. That is why, for the purpose of illustration in this work, only corrosion of the longitudinal girders is considered. Both longitudinal and shear reinforcement of the longitudinal girders are considered to be subjected to corrosion. Hence, the shear failure and bending failure as given by Equation (16) and Equation (17) are considered as limit states (CEN, 2005). The distributions of the stochastic variables are given in Table 8. The characteristic traffic loads are defined according to (CEN, 2003). The failure probabilities are calculated for the most critical section with the cross-section from Figure 7 (b). Since the bridge is considered isostatic and simply supported at the abutments, the critical section for the bending limit state will be close to midspan and the critical section for the shear limit state will be close to the supports. Due to the spatial variability, it is difficult to assign the exact failure location on beforehand, since this might vary depending on the location of 
the region with the lowest resistance. The failure probability is evaluated as the maximum of the shear and bending failure probability, according to (Estes \& Frangopol, 1999). The correlation between the two limit states is also evaluated using the vectors of the sensitivities, but this correlation is found to be small:

$$
\begin{gathered}
g(\boldsymbol{X}, t)=K_{R, M} A_{s}(t) f_{y}\left(h-c-0,5 \frac{A_{s}(t) f_{y}}{b f_{c}}\right)-K_{E, M}\left(M_{G}+M_{Q}\right) \\
g(\boldsymbol{X}, t)=K_{R, V}\left[\left[0.18 k\left(100 \rho_{l} f_{c}\right)^{\frac{1}{3}}\right] b_{w} d+\frac{A_{s w}}{s} z f_{y} \cot (\theta)\right]-K_{E, V}\left(V_{G}+V_{Q}\right)
\end{gathered}
$$

For the loads, a uniform load of $1.2 \mathrm{kN} / \mathrm{m}^{2}$ is applied to represent the permanent loads of the pedestrian pavement. This is applied over the outer $4.5 \mathrm{~m}$ of the bridge deck. The permanent load of the road equals $3.9 \mathrm{kN} / \mathrm{m}^{2}$ and is applied to the part where the actual driveway for the cars is present. The load for proof-loading is based on models retrieved from (Ministry of Public Works Flanders (MOW), personal communication, September 17, 2020) and consists of loaded surfaces of $2.33 \mathrm{~m}$ by $0.5 \mathrm{~m}$ with a load of $84.35 \mathrm{kN} / \mathrm{m}^{2}$, representing trucks driving over the bridge. To have a large enough proof-load, not exceeding the cracking load (i.e. there is no transition to the cracked state and linear elastic behaviour can still be assumed), a number of trucks are placed on the different lanes of the bridge.

The corrosion variables and their parameters (mean and standard deviation) are considered the same as for the illustrative example. For each of the longitudinal girders, an independent random field is considered. Since from the earlier observations correlation model 2 is found to be the most robust, this model is used as the correlation model for the prior random fields of the initiation period and corrosion rate in this case study. Hence, updating is performed when the prior random fields for the corrosion variables are modelled with model 2 and the corrosion degree of the measurement results is sampled (based on samples of the corrosion rate and initiation period) with each of the other models described above as actual correlation model. The data employed in the updating procedure consists of strains measured at the bottom fibres of the longitudinal girders, at four locations along each girder. The measurement locations are indicated with red circles on Figure 5. A normally distributed measurement error with zero mean and a standard deviation of $5 \mu \varepsilon$ is considered.

Table 8 Distributions used in the evaluation of the limit state equations of the case study

\begin{tabular}{llllll}
\hline Symbol & Unit & Dist. & Mean & Std. & Ref. \\
\hline$K_{R, M}$ & - & $\mathrm{LN}$ & 1.2 & 0.18 & (JCSS, 2001) \\
$A_{s}$ & $\mathrm{~mm}^{2}$ & - & 5026 & - & - \\
$f_{y}$ & $\mathrm{MPa}$ & $\mathrm{LN}$ & 560 & 30 & (Holicky \& Sykora, 2010) \\
$f_{c}$ & $\mathrm{MPa}$ & $\mathrm{LN}$ & 39.1 & 7.03 & (Holicky \& Sykora, 2010) \\
$h$ & $\mathrm{~mm}$ & - & 885 & - & - \\
$c$ & $\mathrm{~mm}$ & - & 40 & - & - \\
$b$ & $\mathrm{~mm}$ & - & 500 & - & - \\
$K_{E, M}$ & - & $\mathrm{LN}$ & 1 & 0.1 & (JCSS, 2001) \\
$M_{G}$ & $\mathrm{Nmm}$ & $\mathrm{N}$ & $\mathrm{G}_{\mathrm{k}}$ & $0.1 \mathrm{G}_{\mathrm{k}}$ & (Holicky \& Sykora, 2010) \\
$M_{Q}$ & $\mathrm{Nmm}$ & $\mathrm{GU}$ & $0.728 \mathrm{Q}_{\mathrm{k}}$ & $0.146 \mathrm{Q}_{\mathrm{k}}$ & (Caspeele, Steenbergen, \& Sykora, 2016) \\
$K_{R, V}$ & - & $\mathrm{LN}$ & 1.4 & 0.35 & (JCSS, 2001) \\
$k$ & - & - & 1.49 & - & - \\
$\rho_{l}$ & - & - & 0.02 & - & - \\
$b_{w}$ & $\mathrm{~mm}$ & - & 2500 & - & - \\
$d$ & $\mathrm{~mm}$ & - & 845 & - & - \\
$K_{E, V}$ & - & $\mathrm{LN}$ & 1 & 0.1 & (JCSS, 2001) \\
$V_{G}$ & $\mathrm{Nmm}$ & $\mathrm{N}$ & $\mathrm{G}_{\mathrm{k}}$ & $0.1 \mathrm{G}_{\mathrm{k}}$ & (Holicky \& Sykora, 2010) \\
$V_{Q}$ & $\mathrm{Nmm}$ & $\mathrm{GU}$ & $0.728 \mathrm{Q}_{\mathrm{k}}$ & $0.146 \mathrm{Q}_{\mathrm{k}}$ & (Caspeele et al., 2016) \\
$A_{s w}$ & $\mathrm{~mm}{ }^{2}$ & - & 157 & - & - \\
$s$ & $\mathrm{~mm}$ & - & 200 & - & - \\
$c o t(\theta)$ & - & - & 1.19 & - & (Ministry of Public Works Flanders (MOW), personal \\
& & & & & communication, September 17, 2020) \\
\hline
\end{tabular}

For comparison, the posterior distribution of the reliability index is also derived assuming the same model as the one used for sampling the measurement result (i.e. the actual model). The distribution of the reliability index found when assuming model 2 is compared with the one assuming the actual model based on which the measurement result is generated. Figure 8 gives the difference in the mean of the reliability index, whereas Figure 9 gives the difference in standard deviation. These results are given relative to the posterior mean reliability index when updating is performed based on the actual correlation model. It can be seen that for all models, a very good approximation of the reliability index is found when updating is performed based on the prior assumption of 
correlation model 2. The maximum deviation in mean reliability index between updating based on model 2 and updating based on the actual model equals $0.77 \%$ and the average deviation equals $0.54 \%$. The maximum deviation on the uncertainty of the reliability index equals $0.66 \%$ and the average deviation equals $0.41 \%$. The largest deviations are found when the actual model is model 5.

The same procedure is also applied assuming model 5 as the prior correlation model in the calibration. This model was found to be performing badly in section 5 in the application to the simply supported beam. This choice is based on the fact that model 5 does not lead to an accurate approximation of the posterior mean reliability index for two out of the seven models (i.e. the dotted line falls out of the boxplot). It could only approximate the posterior distribution of three of the other models to a reasonable extent. The difference in mean of the reliability index for calibration with model 5 compared to the mean of the reliability index assuming the actual correlation model is calculated and the results are summarized in Table 9. On the right of this table, it can be seen that for models 1 to 4, the performance is quite good. However, for models 6 and 7, the approximation is significantly worse. On the left of Table 9, the results are also compared to those when assuming model 2. Here it can be seen that when the prior model is assumed to be model 5 , the following approximations are better compared to assuming model 2 a priori: the standard deviation of the reliability index when the actual model is model 1, the mean of the reliability index when the actual model is model 4 and both the mean and standard deviation of the reliability index when the actual model is model 3.

Following approximations are worse when assuming model 5 a priori instead of model 2: the mean of the reliability index when the actual model is model 1, and in general the performance is significantly worse when the actual model is model 6 or 7 . Hence, this counters the slightly better performance for some of the other models. In general, model 2 has a better performance, inducing no large deviations for all of the other correlation models. Based on the results for this specific situation and the case study just described, it could be concluded that model 2 is a more robust choice for the prior correlation model. When looking at Table 9, following additional conclusions can be made. When considering the deviation in mean, model 2 best approximates the actual model 7, which both have a zero asymptotic value in Figure 1. When the prior model is model 5, considering the mean, models 3 and 4 are best approximated, which are of the same model 'family'.

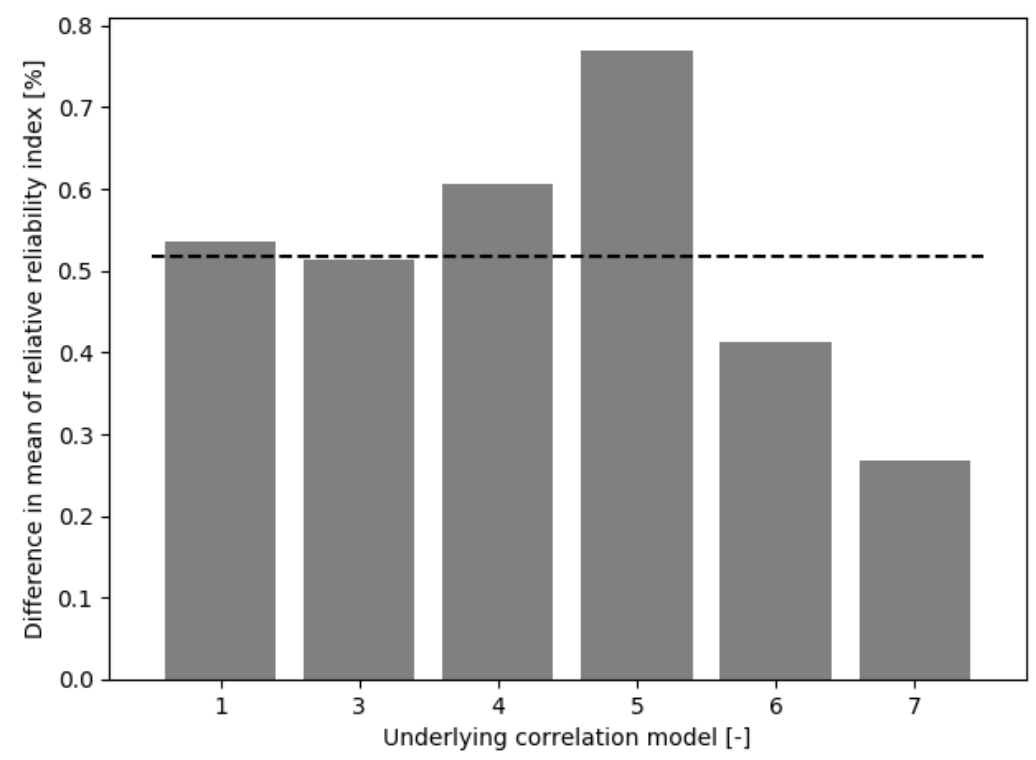

Figure 8 Difference in mean of the reliability index when updated based on correlation model 2 compared to the mean of the reliability index when updated based on the actual underlying correlation model. The dashed line represents the mean of the plotted values. 


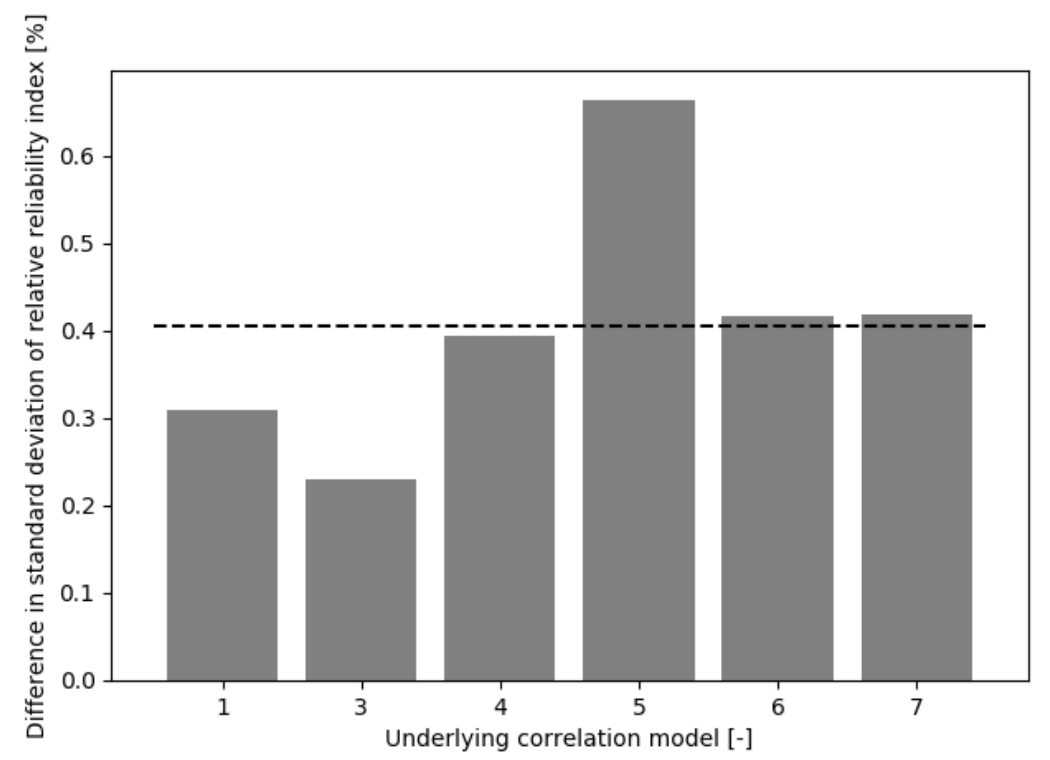

Figure 9 Difference in standard deviation of the reliability index when updated based on correlation model 2 compared to the standard deviation for the reliability index when updated based on the actual underlying correlation model. The dashed line represents the mean of the plotted values.

Table 9 Comparing the performance of model 2 and model 5 for the prior model for the considered RC bridge

\begin{tabular}{c|cc|cc}
\hline \multirow{2}{*}{ Actual model } & \multicolumn{2}{|c|}{ Prior model 2 in calibration } & \multicolumn{2}{c}{ Prior model 5 in calibration } \\
$\boldsymbol{\Delta} \boldsymbol{\mu}_{\boldsymbol{\beta}}[\boldsymbol{\%}]$ & $\boldsymbol{\Delta} \boldsymbol{\sigma}_{\boldsymbol{\beta}}[\boldsymbol{\%}]$ & $\boldsymbol{\Delta} \boldsymbol{\mu}_{\boldsymbol{\beta}}[\boldsymbol{\%}]$ & $\boldsymbol{\Delta} \boldsymbol{\sigma}_{\boldsymbol{\beta}}[\boldsymbol{\%}]$ \\
\hline 1 & 0.54 & 0.31 & 0.92 & 0.12 \\
2 & 0 & 0 & 0.62 & 0.39 \\
3 & 0.51 & 0.23 & 0.44 & 0.05 \\
4 & 0.61 & 0.39 & 0.30 & 0.35 \\
5 & 0.77 & 0.66 & 0 & 0 \\
6 & 0.41 & 0.42 & 59.5 & 166 \\
7 & 0.27 & 0.42 & 105 & 163 \\
\hline
\end{tabular}

The reliability index of the bridge is also calculated based on deterministic assumptions on the mean and standard deviation of the initiation period and corrosion rate. The mean and standard deviation of the initiation period are assumed equal to 20 years and 10 years respectively. The mean and standard deviation of the corrosion rate are equal to $0.035 \mathrm{~mm} /$ year and $0.02 \mathrm{~mm} /$ year respectively. This reliability index is calculated for the different correlation models. It is then compared with the mean posterior reliability index calculated based on the same prior correlation model and actual underlying correlation model. On average, the ratio between this mean reliability index and the reliability index based on fixed values for the mean and standard deviation of the initiation period and corrosion rate equals 1.11 .

\section{CONCLUSIONS}

For RC structures subjected to corrosion, the reliability index can be calculated to estimate the remaining service life. To arrive at a more accurate estimate, measurements can be performed. These can include visual observations, static strain or deflection measurements, dynamic tests resulting in modal data, destructive measurements, etc. (Vereecken et al., 2021). In this work, the focus was on static strain data. The resulting data can be used to update the reliability index. In order to perform such Bayesian updating, prior assumptions are required, which might influence the posterior distribution. One of these assumptions concerns the choice of the correlation model for the spatial behaviour of the corrosion variables. Up to date, there is no generally agreed upon correlation model for the spatial variation of corrosion variables, although its choice will affect the resulting reliability index. In this work, the influence of the prior correlation model considered for corrosion variables on the distribution of the reliability index is investigated. For this purpose, a simply supported beam subjected to corrosion is considered. The initiation period and corrosion rate are modelled with random fields and the distributions of their means and variances are updated based on static strain data. These posterior distributions are generated based on an approximate hierarchical Bayesian method. 
In order to investigate the impact of a mismatch between the actual (unknown) correlation of corrosion and the one assumed in the prior models for calibration, the study is performed considering seven different correlation models and/or correlation lengths that are selected from a literature survey. For all these seven sets of data, the posterior distribution of the reliability index is derived for each of the seven prior assumptions on the correlation model. For the investigated illustrative example considering a simply supported beam, a squared exponential model with correlation length $2 \mathrm{~m}$ is found to give posterior distributions of the reliability index close to the actual distribution in almost all seven cases. Hence, in this particular situation, this correlation model might be the best choice when the initiation period and corrosion rate are modelled by random fields. The robust character of this correlation model is confirmed in a case study where the seven selected correlation models are considered for a real bridge geometry.

Some final remarks should be made. First, it should be pointed out that a bad model can lead to results that are more conservative. In estimation problems a minimum variance unbiased solution is typically desired. The bias needs to be as small as possible. When different solutions have the same bias, the one with the minimum variance can be considered. Hence, in this manuscript, the focus is on the bias between the actual posterior mean of the reliability index and the one found when assuming a wrong correlation model. This bias should be minimized by choosing the most appropriate prior correlation model.

Second, it is pointed out here that in reality many other sources of model errors will also be present. A (finite element) model will always lead to a predicted behaviour that differs from the one of the actual structure. This deviation can for example be ascribed to differences in modelled and actual support conditions, idealized material models, etc. All these errors might contribute to a bias between predicted and actual structure. When considering the reliability index, these model errors might have an influence and are often incorporated by a load and/or resistance model error in the limit state function, for example according to the JCSS probabilistic model code (JCSS, 2001). When considering the results of the reliability index in this article, there can indeed be a significant influence of choosing a wrong prior correlation model on the distribution of the reliability index. Hence, this type of model error is relevant to consider as well. An impression on the magnitude of the modelling error can be extracted from the analyses made at the end of sections 5 and 6 . Here the percentages of deviation between different model assumptions are provided. This provides a general idea on the fact that when a more robust correlation model is applied as the prior model, the uncertainties are limited. For a less robust correlation model as the prior model, the uncertainties are larger, represented by the larger percentages of the deviation between the resulting reliability index and the actual reliability index.

\section{ACKNOWLEDGMENTS}

This research has been made possible through funding from the Research Foundation Flanders (FWO) under grant number G013317N, for which FWO is gratefully acknowledged. In addition, Ministry of Public Works Flanders (MOW) is gratefully acknowledged for providing the case study.

\section{REFERENCES}

Beck, J. L., \& Katafygiotis, L. S. (1998). Updating Models and Their Uncertainties. I: Bayesian Statistical Framework. ASCE Journal of Engineering Mechanics, 124(April), 455-461.

Behmanesh, I., Moaveni, B., Lombaert, G., \& Papadimitriou, C. (2015). Hierarchical Bayesian model updating for structural identification. Mechanical Systems and Signal Processing, 64-65, 360-376. https://doi.org/10.1016/j.ymssp.2015.03.026

Björk, A. (1996). Numerical methods for least squares problems. Philadelphia, USA: SIAM Society for Industrial and Applied Mathematics.

Botte, W. (2017). Quantification of Structural Reliability and Robustness of New and Existing Concrete Structures Considering Membrane Action.[Doctoral dissertation, Ghent University]

Bucher, C. (2009). Computational Analysis of Randomness in Structural Mechanics (D. M. Frangopol, ed.). CRC Press.

Caspeele, R., Steenbergen, R., \& Sykora, M. (2016). fib Bulletin 80: Partial factor methods for existing concrete structures.

CEN. (2003). EN 1991-2: Eurocode 1: Actions on structures - Part 2: Traffic loads on bridges.

CEN. (2005). EN 1992-1-1: Eurocode 2: Design of concrete structures - Part 1-1: General rules and rules for buildings.

Criel, P., Caspeele, R., \& Taerwe, L. (2014). Bayesian updated correlation length of spatial concrete properties using limited data. Computers and Concrete, 13(5), 659-677. https://doi.org/10.12989/cac.2014.13.5.659

Der Kiureghian, A. (2008). Analysis of structural reliability under parameter uncertainties. Probabilistic Engineering Mechanics, 23(4), 351-358. https://doi.org/10.1016/j.probengmech.2007.10.011 
Duprat, F. (2007). Reliability of RC beams under chloride-ingress. Construction and Building Materials, 21(8), $1605-1616$.

Duracrete. (2000). DuraCrete - Probabilistic Performance based Durability Design of Concrete Structures. Report No BE9521347.

Engelund, S. (1997). Probabilistic Models and Computational Methods for Chloride Ingress in Concrete. [Doctoral dissertation, Aalborg University]

Enright, M. P., \& Frangopol, D. M. (1999). Condition Prediction of Deteriorating Concrete Bridges Using Bayesian Updating. Journal of Structural Engineering, 125(October), 1118-1125.

Estes, A. C., \& Frangopol, D. M. (1999). Repair Optimization of Highway Bridges Using System Reliability Approach. Journal of Structural Engineering, 125, 766-775.

Faroz, S. A., Pujari, N. N., \& Ghosh, S. (2016). Reliability of a corroded RC beam based on Bayesian updating of the corrosion model. Engineering Structures, 126, 457-468. https://doi.org/10.1016/j.engstruct.2016.08.003

fib. (2006). fib Bulletin 34: Model code for service life design. Lausanne.

Gelman, A., Carlin, J. B., Stern, H. S., \& Rubin, D. B. (2004). Bayesian Data Analysis Texts in Statistical Science. In C. Chatfield, M. Tanner, \& J. Zidek (Eds.), Development (Second, Vol. 134). CHAPMAN \& HALL/CRC.

Geman, S., \& Geman, D. (1984). Stochastic Relaxation, Gibbs Distributions, and the Bayesian Restoration of Images. IEEE Transactions on Pattern Analysis and Machine Intelligence, PAMI-6(6), 721-741. https://doi.org/10.1109/TPAMI.1984.4767596

Hajializadeh, D., Stewart, M. G., Enright, B., \& OBrien, E. (2015). Spatial time-dependent reliability analysis of reinforced concrete slab bridges subject to realistic traffic loading. Structure and Infrastructure Engineering. https://doi.org/10.1080/15732479.2015.1086385

Holicky, M., \& Sykora, M. (2010). Stochastic models in analysis of structural reliability. Proceedings of the International Symposium on Stochastic Models in Reliability Engineering, Life Sciences and Operations Management, pp. 428-439.

Ipsen, I. C. (2009). Numerical matrix analysis. Linear systems and least squares. Philadelphia, PA, USA: SIAM Society for Industrial and Applied Mathematics.

JCSS. (2001). JCSS Probabilistic Modelcode. https://doi.org/10.13109/9783666538452.10

Lay, S., Schieß1, P., \& Cairns, J. (2003). Lifecon Deliverable D3.2.

Li, Y., Vrouwenvelder, T., Wijnants, G. H., \& Walraven, J. (2004). Effect of Spatial Variability on Maintenance and Repair Decisions for Concrete Structures. In Structural Concrete (Vol. 5).

Liu, P. L., \& Der Kiureghian, A. (1986). Multivariate distribution models with prescribed marginals and covariances. Probabilistic Engineering Mechanics, 1(2), 105-112. https://doi.org/10.1016/02668920(86)90033-0

Papadimitriou, C., Beck, J., \& Katafygiotis, L. (1997). Asymptotic expansions for reliability and moments of uncertain systems. ASCE Journal of Engineering Mechanics, 123(December), 1219-1229.

Samarakoon, S. M. S. M. K., \& Sælensminde, J. (2015). Condition assessment of reinforced concrete structures subject to chloride ingress: A case study of updating the model prediction considering inspection data. Cement and Concrete Composites, 60, 92-98. https://doi.org/10.1016/j.cemconcomp.2015.03.011

Simoen, E. (2013). Uncertainty Quantification in Finite Element Model Updating [Doctoral dissertation, KU Leuven]

Simoen, E., Papadimitriou, C., \& Lombaert, G. (2013). On prediction error correlation in Bayesian model updating. Journal of Sound and Vibration, 332(18), 4136-4152. https://doi.org/10.1016/j.jsv.2013.03.019

Stewart, M. G. (2006). Spatial variability of damage and expected maintenance costs for deteriorating RC structures. Structure and Infrastructure Engineering, 2(2), 79-90. https://doi.org/10.1080/15732470500253230

Stewart, M. G., \& Mullard, J. A. (2007). Spatial time-dependent reliability analysis of corrosion damage and the timing of first repair for RC structures. Engineering Structures, 29(7), 1457-1464. https://doi.org/10.1016/j.engstruct.2006.09.004

Stewart, M. G., \& Rosowsky, D. V. (1998). Time-dependent reliability of deteriorating reinforced concrete bridge decks. Structural Safety, 20(1), 91-109. https://doi.org/10.1016/S0167-4730(97)00021-0

Stewart, M. G., \& Suo, Q. (2009). Extent of spatially variable corrosion damage as an indicator of strength and time-dependent reliability of RC beams. Engineering Structures, 31(1), 198-207. https://doi.org/10.1016/j.engstruct.2008.08.011

Straub, D. (2011). Reliability updating with inspection and monitoring data in deteriorating reinforced concrete slabs. Application of Statistics and Probability in Civil Engineering. London.

Straub, D., Malioka, V., \& Faber, M. H. (2009). A framework for the asset integrity management of large deteriorating concrete structures. Structure and Infrastructure Engineering, 5(3), 199-213. https://doi.org/10.1080/15732470601017369 
Sudret, B., \& Kiureghian, a Der. (2000). Stochastic Finite Element Methods and Reliability: A State-of-the-Art Report. University of California Berkeley, (November), 189

Tran, T. V., Bastidas-Arteaga, E., Schoefs, F., Bonnet, S., O’Connor, A. J., \& Lanata, F. (2012). Structural reliability analysis of deteriorating RC bridges considering spatial variability. Bridge Maintenance, Safety, Management, Resilience and Sustainability - Proceedings of the Sixth International Conference on Bridge Maintenance, Safety and Management, (July), 698-705. https://doi.org/10.1201/b12352-94

Vanmarcke, E. (2010). Random Fields Analysis and Synthesis. World Scientific.

Vereecken, E., Botte, W., Lombaert, G., \& Caspeele, R. (2020). Bayesian decision analysis for the optimization of inspection and repair of spatially degrading concrete structures. Engineering Structures, 220(June), 111028. https://doi.org/10.1016/j.engstruct.2020.111028

Vereecken, E., Botte, W., Lombaert, G., \& Caspeele, R. (2021). A Bayesian inference approach for the updating of spatially distributed corrosion model parameters based on heterogeneous measurement data. Structure and Infrastructure Engineering.

Vu, K. A., \& Stewart, M. G. (2005). Predicting the Likelihood and Extent of Reinforced Concrete CorrosionInduced Cracking. Journal of Structural Engineering, 131(11), 1681-1689. https://doi.org/10.1061/(asce)0733-9445(2005)131:11(1681)

Vu, K. A. T. (2003). Corrosion-induced cracking and spatial timedependent reliability analysis of reinforced concrete structures. The University of Newcastle, New South Wales, Australia.

Ying, L., Vrouwenvelder, T., \& Wijnants, G. H. (2004). Spatial Variability of Concrete Degradation. Life-Cycle Performance of Deteriorating Strucutres: Assessment, Design and Management, 49-58. Retrieved from papers2://publication/uuid/84A331C6-45C3-49E8-A030-CD9A196CD9CB 